\title{
Lethal(2)giant larvae is required in the follicle cells for formation of the initial AP asymmetry and the oocyte polarity during Drosophila oogenesis
}

\author{
Qi Li ${ }^{1, *}$, Tianchi Xin ${ }^{1, *}$, Wenlian Chen ${ }^{1}$, Mingwei Zhu ${ }^{1}$, Mingfa $\mathrm{Li}^{1}$ \\ ${ }^{1}$ Laboratory of Developmental Genetics, Bio-X Life Science Research Center and School of Life Science and Biotechnology, Shanghai \\ Jiao Tong University, Shanghai 200240, China
}

The intricately regulated differentiation of the somatic follicle cell lineages into distinct subpopulations with specific functions plays an essential role in Drosophila egg development. At early oogenesis, induction of the stalk cells generates the first anteroposterior (AP) asymmetry in the egg chamber by inducing the posterior localization of the oocyte. Later, the properly specified posterior follicle cells signal to polarize the oocyte along the AP and dorsoventral (DV) axes at mid-oogenesis. Here, we show that lethal(2)giant larvae (lgl), a Drosophila tumor suppressor gene, is required in the follicle cells for the differentiation of both stalk cells and posterior follicle cells. Loss-of-function mutations in $l g l$ cause oocyte mispositioning in the younger one of the fused chambers, due to lack of the stalk. Removal of $l g l$ function from the posterior follicle cells using the FLP/FRT system results in loss of the oocyte polarity that is elicited by the failure of those posterior cells to differentiate normally. Thus, we provide the first demonstration that $l g l$ is implicated in the formation of the initial AP asymmetry and the patterning of the AP and DV axes in the oocyte by acting in the specification of a subset of somatic follicle cells.

Keywords: Drosophila oogenesis, $l g l$, oocyte positioning, stalk, oocyte polarity, posterior follicle cells, differentiation Cell Research (2008) 18:372-384. doi: 10.1038/cr.2008.25; published online 12 February 2008

\section{Introduction}

Lethal(2)giant larvae ( $(g l)$ is the first tumor suppressor gene identified and molecularly cloned in Drosophila melanogaster $[1,2]$. Mutations in $l g l$ produce a lethal malignant tumor-like phenotype, characterized by loss of cell polarity and uncontrolled proliferation of the epithelium in imaginal discs, as well as of neuroblasts in brain during larvae development [3-6]. Genetic and phenotypic analyses have also implicated $l g l$ in multiple developmental and biological processes, such as oogenesis [7-9], embryogenesis [8], dorsal closure [8, 10] and asymmetric division of neuroblasts $[11,12]$, indicating that $\mathrm{Lgl}$ may act in more cell populations than imaginal discs. In Drosophila oogenesis, the absence of $l g l$ gene function blocks

\footnotetext{
*These two authors contributed equally to this work.

Correspondence: Mingfa Li

Tel: +86-21-34204918; Fax: +86-21-34204192

E-mail: mfli@sjtu.edu.cn

Received 10 December 2007; accepted 20 December 2007; published online 12 February 2008
}

egg development at certain stages, showing a multilayered accumulation of follicular cells at both extremities of egg chambers, fused egg chambers, and apoptosis of germline cells $[8,9]$. However, the precise mechanism by which Lgl functions in oogenesis remains unclear.

Drosophila oogenesis begins with the asymmetric division of the germline stem cells located at the anterior tip of the germarium, which gives rise to one daughter stem cell and one cystoblast. The cystoblast subsequently undergoes four rounds of consecutive cell divisions with incomplete cytokinesis, producing a cyst of 16 interconnected cells. In germarial region $2 b$, one of these germline cells differentiates as oocyte, whereas the remaining 15 become nurse cells. As the cyst moves down the germarium from region $2 \mathrm{~b}$ to region 3 , the oocyte becomes localized to the posterior of the nascent egg chamber through homophilic adhesive interactions with the posterior follicle cells (Figure 1A) $[13,14]$. The posterior positioning of the oocyte generates the initial asymmetry that contributes to the polarization of the anteroposterior (AP) and dorsoventral (DV) axes in the oocyte, and later in the embryo [15].

The Drosophila body axes are established during oo- 
genesis through reciprocal signaling between the oocyte and the surrounding epithelial follicle cells (Figure 1B). First, Gurken (Grk) signals from the posteriorly localized oocyte to activate the epidermal growth factor receptor (EGFR) in the overlying terminal follicle cells, and induces a posterior cell fate during stage 2-6 [16, 17]. Later, at stage 7-10, these specified posterior follicle cells send back an unidentified signal to the oocyte, resulting in the reorganization of its microtubule (MT) cytoskeleton [18-20]. This directs the MT-dependent localization of bicoid $(b c d)$ and oskar (osk) mRNA to the anterior and posterior pole of the oocyte, respectively, thus defining the AP polarity of the oocyte, and the future AP axis of the embryo [21-24]. The repolarization of the MT cytoskeleton also causes the migration of the oocyte nucleus from the posterior to the anterior cortex and its anchoring at the dorsal anterior corner. At this time, the localized Grk around the nucleus signals for a second time to induce the overlying main body follicle cells to adopt a dorsal cell fate, specifying the pattern of the DV axis $[25,26]$. So far, the molecular nature of the hypothetical posterior follicle cell signal has remained elusive. Nevertheless, an increasing number of factors have been identified in both the posterior follicle cells and the oocyte that are required for the generation and transduction of this unknown signal [27-31].

The Drosophila egg development requires precisely coordinated activities of both germline cells and associated somatic follicle cells. The proper differentiation of the follicle cells into several distinct subpopulations with specific functions is essential for egg development. The follicle stem cells reside in region $2 b$ of the germarium and give rise to two distinct lineages: the epithelial cell precursors

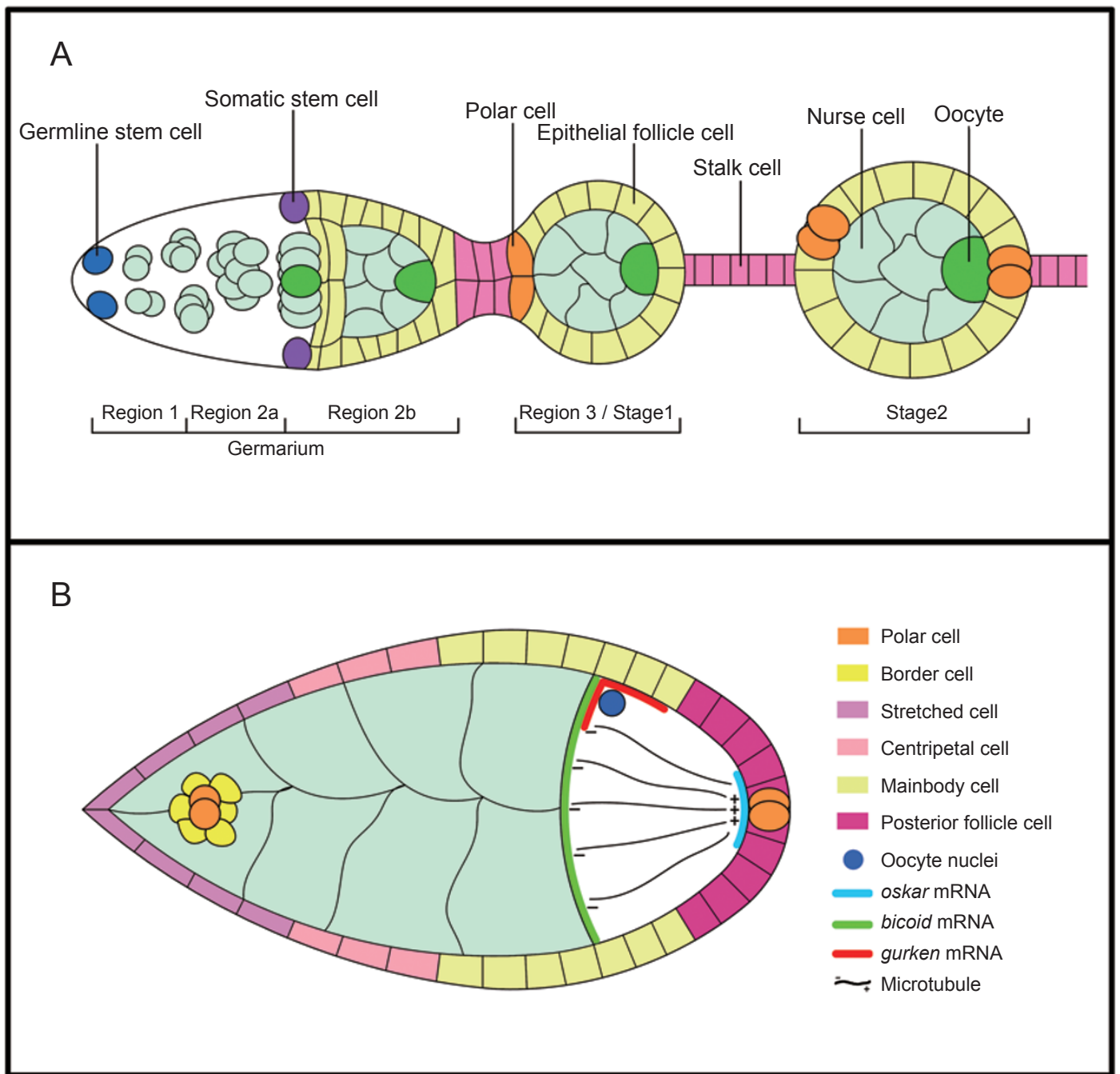

Figure 1 Schematic representation of early oogenesis (A) and stage 9 egg chamber (B). The germline and specified somatic cells are indicated individually. In both panels, anterior is to the left. 
and the polar/stalk cell precursors. The former generates a monolayer epithelium that surrounds each cyst in the germarium and continues to proliferate until stage 6 . As the cyst becomes enveloped by the epithelial follicle cells, pinching from the germarium, the polar/stalk cell lineage cease their divisions, differentiate into one pair of polar cells at each pole of the egg chamber and the stalk cells that form a bridge between adjoining chambers (Figure 1A) [32, 33]. Studies on genetic control of the polar/stalk cell differentiation have led to a model explaining formation of the initial AP asymmetry in Drosophila egg development [34]. In this model, each cyst determines the posterior positioning of the oocyte and the subsequent polarization of the AP axis in the following younger cyst via a relay mechanism that involves a series of posterior to anterior inductions. Later, the follicular epithelium differentiates into five epithelial fates: border, stretched, centripetal, posterior, and main body cells during mid-oogenesis (Figure 1B). From stage 9 onwards, the differentiated follicle cells express fate-specific markers, and undergo appropriate migrations and morphological changes. Each of these subpopulations exerts a specific function in the production of a mature egg, and proper AP axial patterning as well [35].

Loss-of-function mutations in $\mathrm{lgl}$ cause the failure of the females to lay eggs $[7,8]$, suggesting an essential role of this gene in oogenesis. To clarify how it functions in this developmental process, we therefore examined the potential involvement of $\mathrm{Lgl}$ in distinct key steps that are crucial for egg development. Here we show that $l g l$ is required in the follicle cells for the posterior positioning of the oocyte in early oogenesis, and for the AP and DV axial patterning at mid-oogenesis. Further, we find that Lgl is implicated in the cell fate specification of a subset of somatic stem cell-derived cell subpopulations, leading to the establishment of the initial AP asymmetry within the egg chamber and the polarization of the oocyte. Thus, we provide more insights into the mechanisms by which $l g l$ functions in Drosophila oogenesis.

\section{Results}

Mutations in $\operatorname{lgl}$ cause oocyte mispositioning in the egg chamber

The posterior positioning of the oocyte is the critical initial step in establishing the polarity of oocyte itself, and later the AP axis in embryo [15]. We therefore wish to investigate whether $\mathrm{Lgl}$ is required for this process in early oogenesis. To this end, we performed immunofluorescence on ovaries from $l g l^{s 3} / \lg l^{4}$ females transheterozygous for the temperature-sensitive allele $\lg l^{t s 3}$ and the null allele $\lg l^{4}$ that were grown at $29^{\circ} \mathrm{C}$ for 1 or 3 days. As previously described, we found that $l g l$ mutation blocks egg chamber maturation at the onset of vitellogenesis with two characteristic phenotypes: follicle cell accumulation at the two terminals of follicles and fusion between adjoining chambers. The penetrance of both phenotypes seen in mutant ovaries appears to increase with the duration of rearing the females at the restrictive temperature. Antibody staining of the ovaries against oo18 RNA-binding protein (Orb), a specific marker for oocyte [36], revealed that in $55 \%$ $(120 / 218)$ of the ovarioles from $\lg l^{t s 3} / \lg l^{4}$ females grown at $29^{\circ} \mathrm{C}$ for 1 day there is one or more oocyte mislocalized from the posterior to a lateral, anterior or central position in the egg chambers (Figure 2A-2C). Out of 218 ovarioles, $133(61 \%)$ displayed a fusion between adjacent chambers, while the remaining 85 had visible stalk that separates the follicles. Coincidentally, there were mispositioned oocytes in the majority $(80 \%, 107 / 133)$ of those ovarioles with fused chambers (Figure 2B). Conversely, the misplaced oocyte appeared in less than $16 \%(13 / 85)$ of the ovarioles without the fusion phenotype (Figure 2C). As expected, when the mutant females were maintained at $29^{\circ} \mathrm{C}$ for 3 days, the penetrance of both oocyte mispositioning and fusion phenotype significantly rose (data not shown). Collectively, these data indicate that $l g l$ is required for oocyte positioning, and suggest that egg chamber fusion is closely correlated with the oocyte mispositioning in the absence of $\lg l$ function.

Lgl is ubiquitously expressed in both germline cells and surrounding follicle cells during oogenesis [37]. It would be important to determine the role of $l g l$ activity from either population of cells in regulating oocyte positioning. For this purpose, we generated $\lg l^{4}$ homozygous clones, either in germline or in follicle cells or in both by temporally heat shocking the FLP/FRT flies [38]. While germline clones were induced during 3rd instar larval stage, heat shocking pupae or adults allowed us to produce somatic clones in the entire follicle cell layer or the restricted compartments of the epithelium. No mispositioned oocytes were observed when only the germ line was completely mutant $(n=131$, Figure 2D), indicating that correct positioning of the oocyte does not depend on germline $\lg l$ activity. By contrast, we found mispositioned oocytes in a number of egg chambers with mosaic follicle cell epithelia in the presence of germline Lgl, implying this gene is required in the follicle cells to regulate the posterior localization of the oocyte. Consistent with the observations in $l g l^{l s 3} / \lg l^{4}$ background, a fusion was routinely seen when $l g l$ was mutated at the posterior/anterior compartment between the adjoining chambers (Figure 2E). Furthermore, a certain percentage (49\%, $65 / 132$ ) of egg chambers with the fusion phenotype showed a misplaced oocyte (Figure 2E). Interestingly, the oocyte mispositioning preferentially occurred in the younger one of the fused chambers $(84 \%, 55 / 65$, arrow in Figure 2E). 

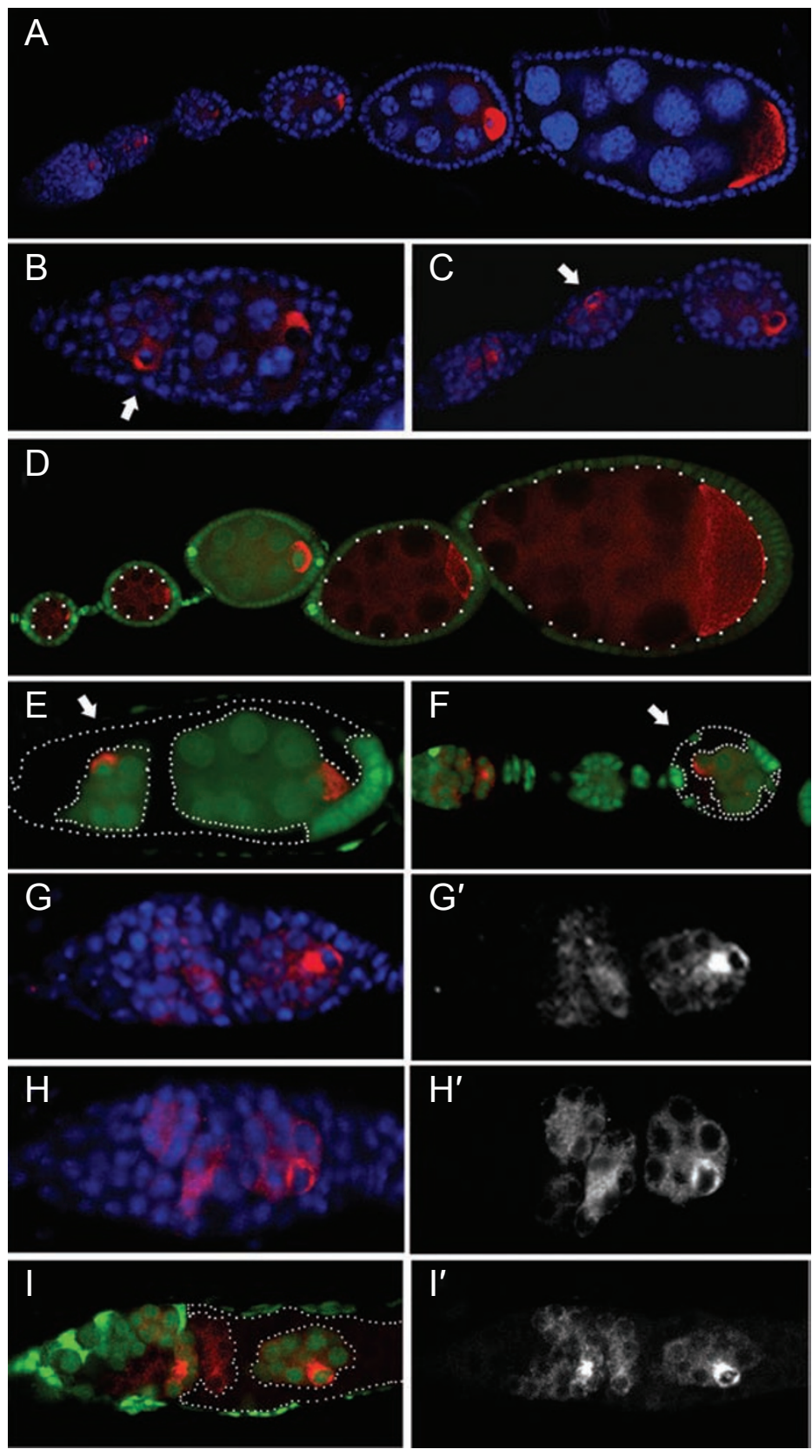

Figure 2 Somatic $\mathrm{Lgl}$ is required for the oocyte positioning at the posterior of egg chambers. (A-C) A wild-type ovariole (A) or $|g|^{t s 3} /|g|^{4}$ egg chambers $(B, C)$ stained for Orb (red) and nuclei (DAPI, blue). In the wild type, oocyte is localized at the posterior of the egg chambers (A). When $\mathrm{lgl}$ is mutated, the oocyte becomes mislocalized in fused (B) or non-fused chambers (arrow in C). Note the oocyte mispositioning in the younger one of the fused chambers (arrow in B). (D-F) Egg chambers bearing $/ g /{ }^{4}$ germline (D) or somatic clones (E, F) marked by the absence of nuclear GFP (green), stained for Orb (red). Oocyte is correctly positioned in the chambers with germline clones (D), but becomes mispositioned when the follicle cell clones cause egg chamber fusion (E) or cover the most part of the epithelium in non-fused chambers (arrow in F). Note the misplaced oocyte in the younger one of the fused chambers (arrow in E). (G-I, G'-I') A wild-type (G, G'), $|g|^{t s 3} /|g|^{4}$ $\left(\mathrm{H}, \mathrm{H}^{\prime}\right)$ or $/ g l^{4}$ mosaic $\left(\mathrm{I}, \mathrm{I}^{\prime}\right)$ germarium stained for Orb (red in $\mathrm{G}-\mathrm{I}$, white in $\mathrm{G}^{\prime}-\mathrm{I}$ ) and nuclei (DAPI, blue in $\mathrm{G}, \mathrm{H}$ ). The $\lg \mathrm{I}^{4}$ clone is marked by the absence of nuclear GFP (green in I). In germarial region 3, the oocyte is normally localized to the posterior of the germline cysts in the wild type (G, $\left.\mathrm{G}^{\prime}\right)$, but mislocalized in $/ g l^{t s} / / g l^{4}\left(\mathrm{H}, \mathrm{H}^{\prime}\right)$ or $/ g l^{4}$ mosaic with a complete follicle cell clone (I, I'). In all panels, anterior is to the left and clones are outlined with dots.
Thus, these data provided more evidence indicative of the correlation between the egg chamber fusions and the oocyte mispositioning observed in $l g l$ mutants.

We next investigated the developmental period at which $l g l$ functions in oocyte positioning by examining the localization of oocyte in the germarium of the mutant ovarioles (Figure 2G-2I). 32\% (20/62) of the germaria from $l g^{l t s 3} / l g l^{4}$ females maintained at $29^{\circ} \mathrm{C}$ for 1 day contained mispositioned oocytes in region 3 (Figure $2 \mathrm{H}$ and $2 \mathrm{H}^{\prime}$ ). A similar phenotype was observed in region 3 of the germarium in which the entire follicle cell epithelium was mutant (43\%, $19 / 44$, Figure $2 \mathrm{I}$ and $\left.2 \mathrm{I}^{\prime}\right)$. Taken together, these data suggest that $l g l$ is required for the initial positioning of the oocyte, rather just for its maintenance at the posterior of developing egg chambers. A similar case has been described in another mutant elsewhere [39].

Lgl is required for the stalk cell differentiation linked to the oocyte positioning in the anterior adjacent egg chamber

Given that the link between fusions of egg chambers and alterations in the specification of stalk cell fate has been described in several mutants [18, 34, 40, 41], we sought to analyze stalk formation in the mutant ovaries using specific antibody and the enhancer trap marker, respectively. 
Antibodies against the stalk marker Big brain (Bib) [42] were employed to assay the stalk cell fate (Figure 3A-3C). No visible stalk cell was detected in an overwhelmingly high percentage $(96 \%, 73 / 76$, Figure $3 \mathrm{~B})$ of the examined fused chambers in ovaries from $l g l^{t s 3} / \lg l^{4}$ females reared at $29^{\circ} \mathrm{C}$ for 1 day, whereas the neighbouring chambers in the same ovariole that were distinctly separated typically contained several Bib-staining cells (Figure 3B). Loss of stalk cell differentiation was also found in the fused chambers with a large clone of $l g l$ mutant follicle cells abutting the two adjacent chambers $(97 \%, 62 / 64$, Figure $3 \mathrm{C}$ and $3 \mathrm{C}^{\prime}$ ). To verify this observation, the expression of $93 \mathrm{~F}$ enhancer trap marker [18] that is specifically expressed in stalk cells of the vitellarium was examined in the mutant ovaries (Figure 3D and 3E). Consistent with the results observed in Bib staining, mutation in $l g l$ leads to loss of the stalk cells $\left(80 / 80\right.$, Figure $3 \mathrm{E}$ and $\left.3 \mathrm{E}^{\prime}\right)$. Altogether, our data demonstrate that inactivation of Lgl perturbs the stalk cell fate determination.

It has been demonstrated that the stalk is required for the proper oocyte positioning in the next younger chamber at its anterior [34]. In our experiments, the misplaced oocyte preferentially appeared in the younger one of the fused chambers in ovaries from either $l g l^{t 53} / l g l^{4}$ females grown at $29{ }^{\circ} \mathrm{C}$ for 1 day $(91 \%, 116 / 127$, arrow in Figure 2B) or females bearing somatic clones homozygous for $l g l^{4}(84 \%$, 55/65, arrow in Figure 2E). A stalk fails to form between almost all the fused chambers, as indicated by the absence of either Bib or $93 \mathrm{~F}$ expression. This phenotype prompted us to infer that loss-of-function mutations in $l g l$ cause the failure of the stalk cell differentiation that consequently leads to oocyte mispositioning.

The data above showed that Lgl functions in oocyte positioning most probably by acting in stalk cell fate specification. It is possible, however, that there exist distinct underlying mechanisms, as a mispositioned oocyte was also detected in a proportion of non-fused chambers in ovaries from either $l g l^{t s 3} / l g l^{4}$ females or the females bearing somatic clones homozygous for $\operatorname{lgl}^{4}$ (Figure 2C and 2F). Mutations in several genes, such as those in the spindle class, seem to affect the proper localization of oocyte by indirectly disrupting the determination and differentiation of the oocyte [43]. Other genes encoding DE-cadherin (DE-cad) and Armadillo (Arm) determine the oocyte positioning through direct regulation of the adhesion of the oocyte to the follicle cells at the posterior of the egg chamber $[13,14]$. To test whether these are affected in $l g l$ mutants, we generated germline clones homozygous for $\lg l^{4}$ and examined the expression of oocyte specification markers Orb, Dynein heavy chain 64C (DHC64C) and Egalitarian (Egl) in the germarium [36, 44, 45]. Similar to the wild type, all these proteins mainly accumulated in the oocyte in germarial region 3 (Figure 4A-4C, data not shown), implying that $\mathrm{Lgl}$ plays no direct role in oocyte differentiation. In contrast, antibody staining revealed that DE-cad or Arm was no longer enriched at the boundary between the oocyte and the posterior follicle cells in region 3 if all the encapsulating epithelial follicle cells were mutant for $\operatorname{lgl}$ (27/38 for Arm, Figure 4D and 4E, data not shown). Instead, the expression of each component of this adhesion complex was randomly clustered on the cell membranes. Thus, our data favor the notion that Lgl can alternatively function in the correct oocyte positioning in newly formed chambers by affecting the adhesion of the oocyte to the posterior of the cyst.

In sum, $\mathrm{Lgl}$ is required for the posterior localization of oocyte in egg chambers primarily by acting in stalk cell differentiation in early oogenesis. In addition, Lgl may execute its function through affecting the DE-cad-mediated adhesion between oocyte and posterior follicle cells.

\section{Lgl is required in the posterior follicle cells for the proper} localization of oocyte polarity determinants

Mutations in $\lg l$ lead to loss of apical-basal polarity in cells of embryonic, imaginal disc and follicular epithelia in Drosophila [5]. In addition, Lgl is required for the recruitment of the cell fate determinants to the basal cell cortex of dividing neuroblasts to ensure asymmetric cell division $[11,12]$. To test the possibility that $\mathrm{Lgl}$ is also implicated the polarization of AP and DV axes in the oocyte at midoogenesis, we generated mosaic egg chambers with clones homozygous for $\mathrm{lgl}^{4}$ allele in either germline or follicle cells, and examined the localization of Staufen (Stau), Osk and Grk, three oocyte polarity determinants, by immunofluoresence [46-48]. In our experiments, we observed that the mosaic chambers with misplaced oocytes can hardly survive beyond the vitellogenesis, which is consistent with the observations in $\lg l^{t s 3}$ mutants $[8,9]$. Therefore, this enabled us to focus our analysis on stage 8-10 mosaic chambers with correctly localized oocytes.

Stau, an RNA-binding protein, colocalizes with osk mRNA throughout oogenesis. At stage 8, Stau along with osk mRNA accumulate transiently at the anterior margins of the oocyte and begin the movement to the posterior pole. These two determinants are predominantly enriched and anchored at the posterior pole during stage 9 and 10, specifying the AP axial patterning of the developing oocyte (Figure $5 \mathrm{~A})$. The expression pattern of Stau remained unchanged in all the examined stage 9/10 egg chambers bearing only germline $l g l$ clones $(\mathrm{n}=133$, Figure $5 \mathrm{~B})$, implying that the germline $\mathrm{Lgl}$ is not required for the localization of this posterior determinant. Conversely, in all the stage 9/10 egg chambers with follicle cell clones covering the posterior terminal $(n=106)$, Stau was no longer concentrated at the 

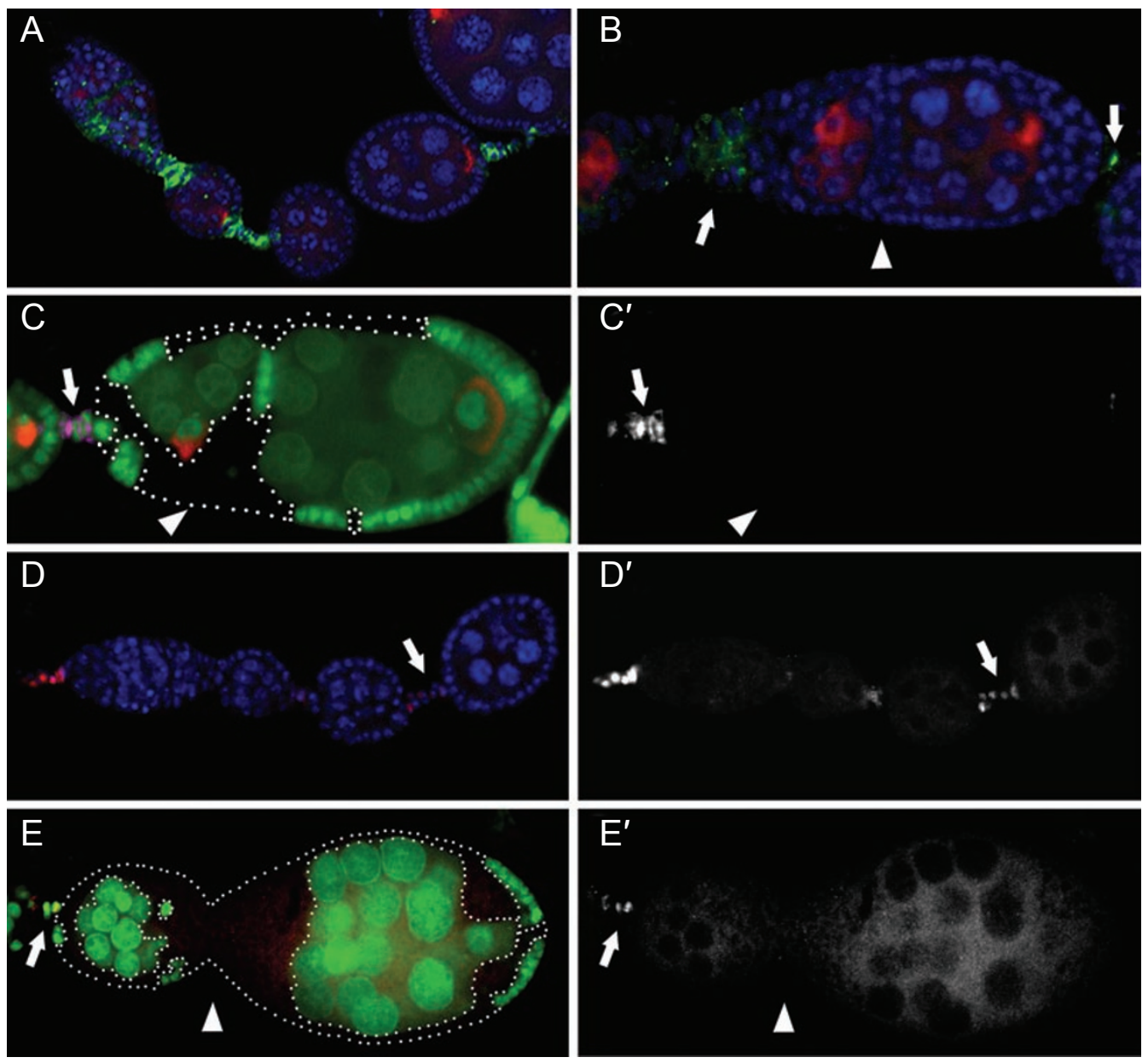

Figure $\mathbf{3} \mathrm{lg} /$ function is essential for the stalk formation linked to the oocyte positioning in the anterior adjacent chamber. (A-C, $\mathrm{C}^{\prime}$ ) A wild-type ovariole (A) or $|g|^{t s 3} / /\left.g\right|^{4}(\mathrm{~B}),\left.\lg \right|^{4}$ mosaic $(\mathrm{C})$ egg chambers stained for Orb (red), Bib (green in A, B, purple in $\mathrm{C}$, white in $\mathrm{C}^{\prime}$ ) and nuclei (DAPI, blue in A, B). The $\mathrm{lg} \mathrm{I}^{4}$ clones are marked by the absence of nuclear GFP (green in C). Bib is expressed in the stalk/polar cell precursors as well as the mature stalk cells in the wild type (A), but is undetectable between the fused chambers induced by Igl mutation (arrowhead in $B, C, C^{\prime}$ ), indicating a loss of stalk cell fate. Note the presence of the stalk between the adjacent non-fused chambers (arrow in B, C, C'). (D, E, D', E') A wild-type ovariole (D) or fused egg chambers containing $/ g l^{4}$ follicle cell clones marked by the absence of nuclear GFP (green), stained for $\beta$-Gal (red in D, E, white in D', $E^{\prime}$ ) and nuclei (DAPI, blue in D). In wild-type ovarioles, the expression of the 93F enhancer trap marker can be observed in all mature stalk cells (arrow in D, D'). However, no 93F-positive cells reside between the two fused chambers (arrowhead in E, E'), also showing a stalkless phenotype caused by mutation in $/ g l$. Note the presence of the stalk between the adjacent non-fused chambers (arrow in E, E'). In all panels, anterior is to the left and clones are outlined with dots.

posterior pole. Instead, it was mislocalized to the center as a dot $(18 \%, 19 / 106$, Figure $5 \mathrm{C})$ or diffusely localized throughout the oocyte $(65 \%, 69 / 106$, data not shown). We also observed a milder phenotype, showing a combined mislocalization of Stau as a central dot plus signal in oocyte cortex (17\%, 18/106, data not shown). As osk mRNA is translated upon its posterior localization at stage 9, we used the anti-Osk antibody staining for tracking osk mRNA localization. Consistent with the observations in Stau expression pattern, while Osk was posteriorly localized in the stage 10 chambers with only germline clones (data not shown), mislocalization of Osk was detected in all the examined stage 10 egg chambers harboring the posterior follicle cell clones ( $n=48$, data not shown). Noticeably, localization of Stau or Osk was never affected in the stage $9 / 10$ mosaic chambers in which the $l g l$ follicle cell clones were only located at the anterior and/or lateral part (data not shown). Taken together, our data showed that $\mathrm{Lgl}$ is required in the posterior follicle cells for the posterior localization of those determinants, and hence for polarization of the AP axis in the oocyte.

Relocalization of grk mRNA and Grk protein accom- 

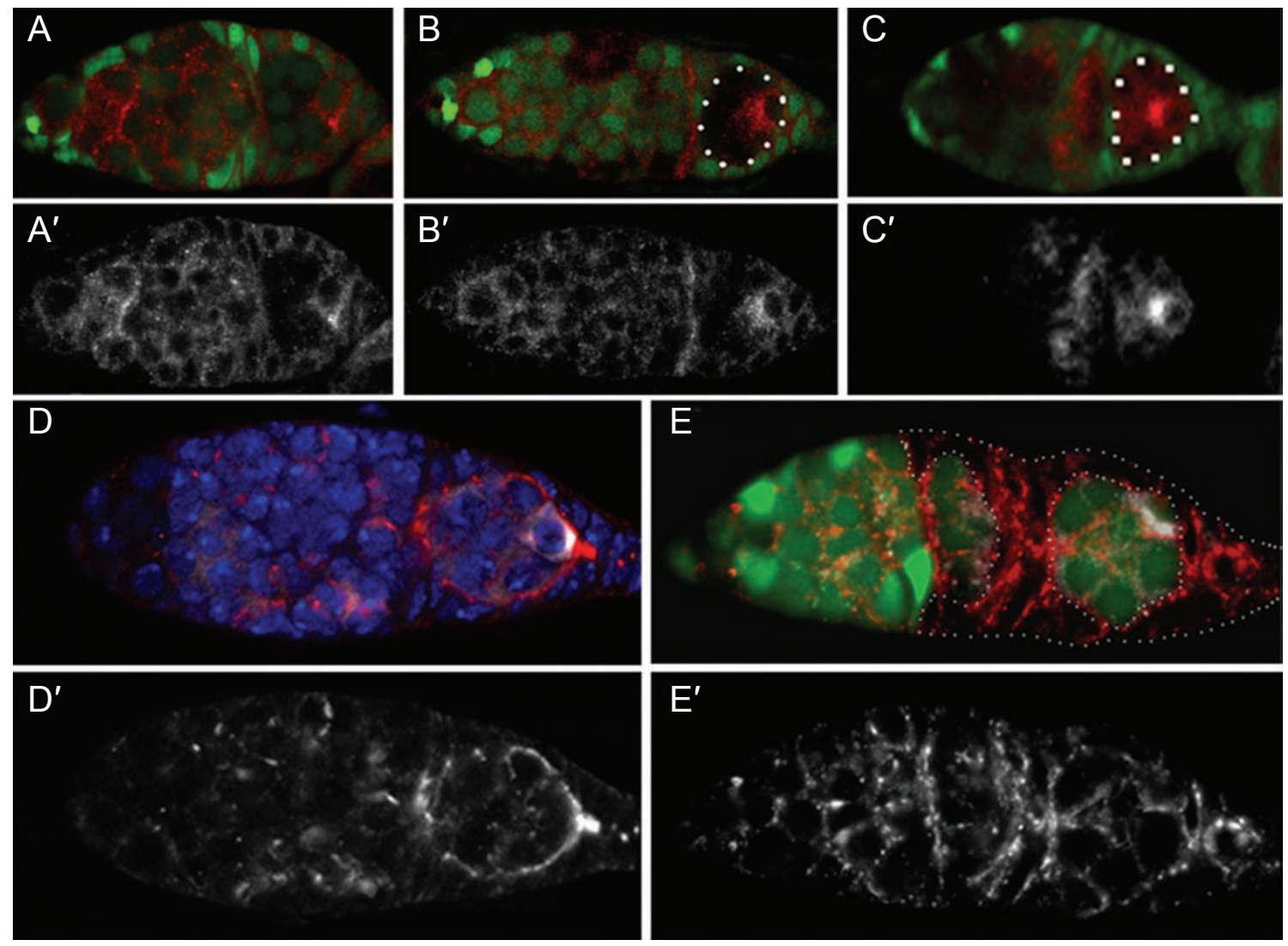

Figure 4 Loss of $/ g /$ function affects the DE-cad complex-mediated adhesion of the oocyte to the overlying follicle cells, but not the oocyte fate determination. (A-C, $\left.A^{\prime}-C^{\prime}\right)$ A wild-type $\left(A, A^{\prime}\right)$ or $I g l^{4}$ mosaic $\left(B, B^{\prime}, C, C^{\prime}\right)$ germarium stained for $D H C 64 C$ (red in $A, B$, white in $A^{\prime}, B^{\prime}$ ) or Orb (red in $C$, white in $\left.C^{\prime}\right)$. The mutant clone is marked by the absence of nuclear GFP (green). As in the wild-type $\left(A, A^{\prime}\right)$, DHC64C or Orb accumulates correctly in the oocyte mutant for $l g l$ in region 3 of the germaria (B, B, C, $\left.C^{\prime}\right)$, indicating a normal specification of the oocyte in Igl germline clones. (D, E, D', E') A wild-type (D, D') or mosaic germarium containing an entire follicle cell clone mutant for $\lg l^{4}\left(\mathrm{E}, \mathrm{E}^{\prime}\right)$ marked by the absence of nuclear GFP (green), stained for Egl (white in D, E), Arm (red in D, E, white in $D^{\prime}, E^{\prime}$ ) and nuclei (DAPI, blue in D). In the wild type, Arm concentrates at the interface between the oocyte and the posterior follicle cells in region $3\left(D, D^{\prime}\right)$. Note the failure of Arm accumulation and the randomly positioned oocyte, due to the absence of $/ g /$ function in the follicle cells (E, E'). In all panels, anterior is to the left and clones are outlined with dots.

panied by the oocyte nucleus migration from the posterior to a point at the anterior margin during mid-oogenesis establishes the DV polarity in the egg chamber, and the future DV axis in the embryo [49]. To test whether Lgl plays a role in this process, we analyzed the distribution of Grk in $l g l$ mutant chambers (Figure 5E-5H). Instead of the dorsal anterior localization in the wild type, Grk was still anchored in the posterior of the oocyte in $71 \%(\mathrm{n}=$ 84 ) of the stage 8-10 egg chambers containing the posterior follicle cell clones (Figure 5G). Consistently, the nucleus failed to migrate from the posterior to the presumptive dorsal anterior corner in the examined cases above (arrowhead in Figure 5G). This phenotype also occurred in the ovaries from $l g l^{t s 3} / l g l^{4}$ females reared at $29^{\circ} \mathrm{C}$ for 1 day $(50 \%, 92 / 182$, Figure $5 \mathrm{~F})$. Similarly, we did not observe any defect in relocalization of Grk in the egg chambers bearing either only germline clones ( $\mathrm{n}=46$, data not shown) or clones located within the anterior/lateral follicle cells (n $=55$, Figure $5 \mathrm{H}$ ). Thus, we find that $\mathrm{Lgl}$ in the posterior follicle cells is also essential for the establishment of DV polarity at mid-oogenesis.

The restricted localization of the determinants above is MT-dependent. Therefore, it is conceivable that inactivation of $\mathrm{Lgl}$ in the posterior follicle cells could result in a failure to repolarize the oocyte MT cytoskeleton as well. To determine whether it is the case, we genetically incorporated the Kin-lacZ transgene [50] into the $l g l^{4}$ FLP/FRT strain to examine the MT organization in the mosaic chambers using the Kinesin- $\beta$-galactosidase (Kin- $\beta$-Gal) fusion as a polarity marker. In the wild type, this fusion protein is localized to the plus ends of the MTs at the posterior pole of the oocyte during stage 8 and 9 (Figure 5I). We found 

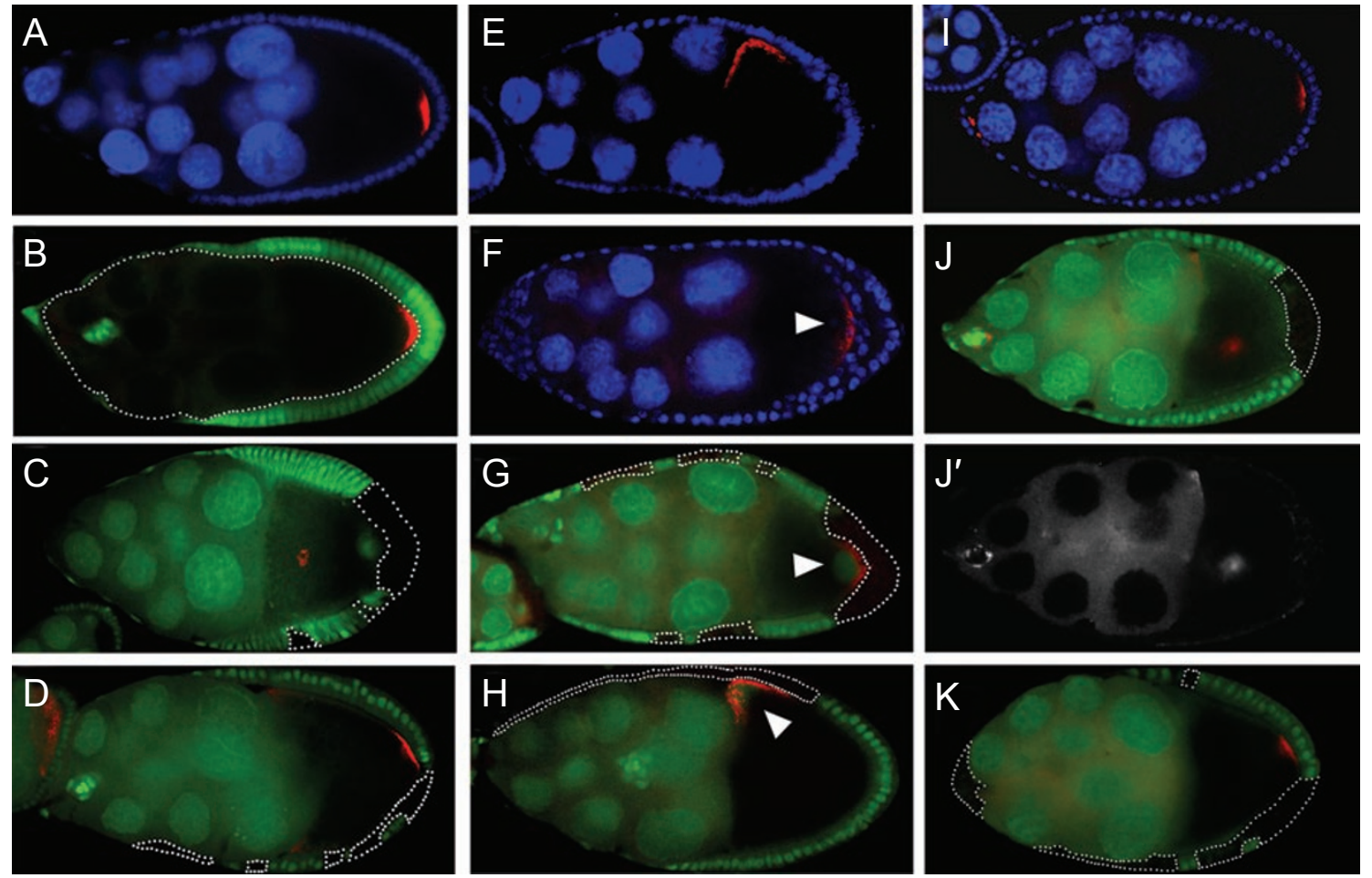

Figure 5 Loss of $/ g /$ function in the posterior follicle cells causes defects in oocyte polarity at mid-oogenesis. (A-D) A stage 9 wildtype (A) or mosaic egg chamber with either a $/ g l^{4}$ germline (B) or a posterior follicle cell clone (C, D) marked by the absence of nuclear GFP (green), stained for Stau (red) and nuclei (DAPI, blue in A). In the wild type, Stau accumulates at the posterior of the oocyte at stage 9-10 (A). Stau is localized normally in stage 9 egg chambers containing the germline clone (B), but mislocalized to the center when all the posterior follicle cells are mutant $(C)$. Note that it becomes localized underneath the remaining wild-type cells when the clone covers only part of the posterior terminal (D). (E-H) A stage 9 wild type (E), $\mid g t^{t s 3} / \mathrm{lg}^{4}(\mathrm{~F})$ or the mosaic egg chamber containing a $/ g l^{4}$ posterior $(\mathrm{G})$ or lateral follicle cell clone $(\mathrm{H})$ marked by the absence of nuclear GFP (green), stained for Grk (red) and nuclei (DAPI, blue in E, F). In the wild type, Grk is localized to the dorsal anterior from stage 8 onwards (E). But Grk and the oocyte nucleus (arrowhead in F, G) still remain at the posterior end instead of the dorsal anterior localization in stage $9 \mathrm{~g} \mathrm{l}^{\mathrm{ts}} / \mathrm{Igl}^{4}$ chambers (F) or the mosaic chamber bearing the posterior clone (G). Note that the localization of Grk and the oocyte nucleus (arrowhead in $\mathrm{H}$ ) is unaffected when the clone only covers the lateral follicle cells $(\mathrm{H})$. (I-K, J') A wild-type (I) or $\mathrm{Ig} / 4$ mosaic chamber ( J-K) at stage 9 stained for $\beta-G a l$ (red in I-K, white in $\mathrm{J}$ ) and nuclei (DAPI, blue in I). Clones are marked by the absence of the nuclear GFP (green in J, K). In the wild type, Kin- $\beta$-Gal is localized at the posterior of the oocyte (I). But the fusion protein is mislocalized to the center when all the posterior follicle cells are mutant $(\mathrm{J}, \mathrm{J}$ ). Note that Kin- $\beta$-Gal becomes localized towards the remaining wild-type cells adjacent to the clone when only a portion of the posterior follicle cells are mutant $(\mathrm{K})$. In all panels, anterior is to the left and clones are outlined with dots. In panels $\mathrm{E}$ and $\mathrm{H}$, dorsal is up.

that Kin- $\beta$-Gal was mislocalized to the center or completely diffused throughout the oocyte in all the examined stage 9 egg chambers harboring a posterior follicle cell clone $(\mathrm{n}=$ 98, Figure 5J and 5J'). DHC64C, another posteriorly localized motor protein at stage 9 , was also mislocalized when the posterior follicle cells were mutant for $l g l$ (data not shown). Thus, we concluded that the absence of $l g l$ function in the posterior follicle cells disrupts the reorganization of the oocyte MT cytoskeleton at mid-oogenesis.

Defective posterior follicle cell differentiation underlies the oocyte polarity phenotype

At mid-oogenesis, a signal delivered by the correctly specified posterior follicle cells triggers the reorganization of the oocyte MT cytoskeleton, leading to the polarization of the AP and DV axes in the oocyte. A number of mutants have been identified that abolish this signal. Among those mutants, many disrupt the specification of the posterior follicle cell fate, rather than the signaling pathway itself $[16,17,51-53]$. As presented above, $\mathrm{Lgl}$ is required in the posterior follicle cells for both the restricted localization of the polarity determinants and the reorganization of the MT cytoskeleton in the oocyte. This prompts us to investigate whether the differentiation of the posterior follicle cells is perturbed in the mosaic chambers showing defects in the polarization of the oocyte. To this end, we stained the ovaries using the antibody against Fas3, which is highly expressed in all immature follicle cells by stage 3 in wild- 
type ovarioles. After that, it is dramatically reduced in epithelial cells, but remains abundantly enriched in pairs of polar cells (Figure 6A). In the mosaic chambers beyond stage 7 with $l g l$ mutant posterior follicle cell clones, we found that Fas 3 expression was highly up-regulated in the majority of those clone cells (Figure 6B), suggesting a defect in their differentiation. In addition, many smaller nuclei were observed in cells of the follicle clones, compared with the polyploid wild-type neighbors, indicating the lack of endocycles in them (Figure 6C). The defective transition from mitosis to endocycle in $l g l$ mutant cells has been described previously [54]. To confirm that the lgl mutant clone cells failed to adopt a posterior fate, a posterior follicle cell marker 998/12 [55] was used in our experiments (Figure $6 \mathrm{D}-6 \mathrm{~F})$. As expected, the expression of this enhancer trap marker was completely absent in the posterior clone cells of stage 8-10 follicles (56/56, Figure 6E). We also observed that the oocyte nuclei remained aberrantly at the posterior in those mosaic chambers, where 998/12 expression was completely missing beyond stage 8 (arrowhead in Figure $6 \mathrm{E}$ ). More interestingly, if the clones only cover part of the posterior follicle cells, the cell fate specification can still occur in the remaining wild-type cells, as indicated by the expression of 998/12 (Figure 6F), suggesting that Lgl acts cell-autonomously in the specification of the posterior cells. This finding is also reflected in our observations that, in stage 9 egg chambers with $l g l^{4}$ clones on only one portion of the posterior end, both Stau and Kin- $\beta$-Gal fusion proteins were mislocalized away from the clones and toward the wild-type cells (Figure 5D and 5K). Taken together, a failure of the posterior follicle cells to differentiate normally is responsible for the observed defects in oocyte polarity at mid-oogenesis.

\section{Discussion}

So far, the role of $\mathrm{Lgl}$ in controlling the apical-basal polarity has been described in multiple epithelial tissues including the follicular epithelium in ovaries, and the neuroblasts as well. In this study, we have demonstrated that $\mathrm{Lgl}$ is also required for the formation of the oocyte polarity along both AP and DV axes in oogenesis. In the former
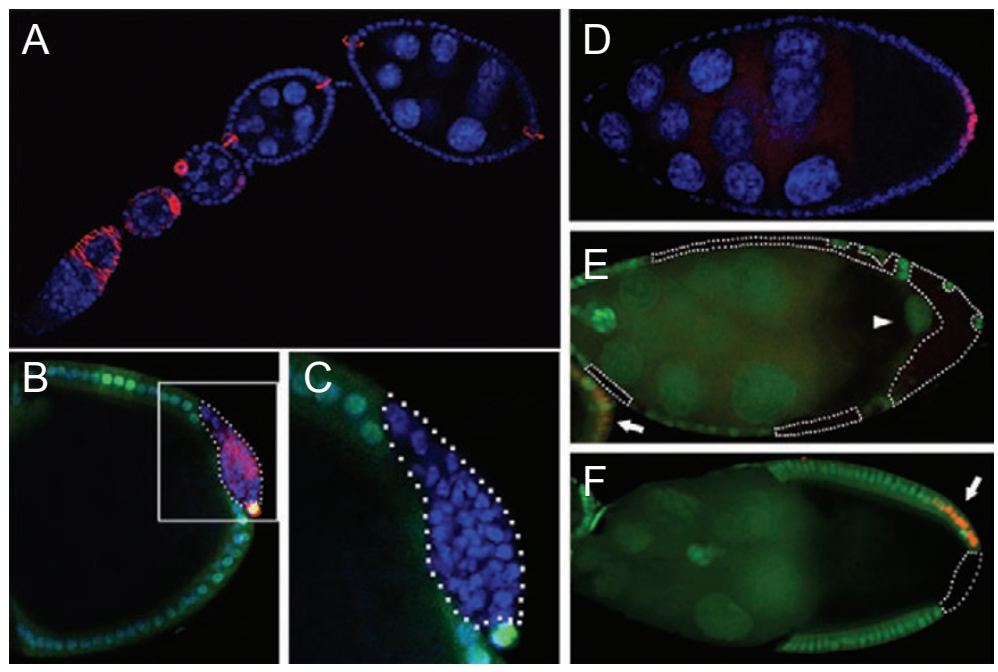

Figure $6 \mathrm{Lgl}$ is required for the differentiation of the posterior follicle cells. (A) A wild-type ovariole stained for Fas3 (red) and nuclei (DAPI, blue). Fas3 is highly expressed in all follicle cells before stage 3 and then soon becomes restricted to the polar cells. (B) A stage 10 egg chamber containing an $/\left.g\right|^{4}$ posterior follicle cell clone marked by the absence of nuclear GFP (green), stained for Fas3 (red) and nuclei (DAPI, blue). The expression of Fas3 is evident in the mutant cells. (C) A magnified view of the boxed region of $(B)$ showing the mutant cells have smaller nuclei (DAPI, blue) than their wild-type neighbors marked by nuclear GFP (green). (D) A wild-type egg chamber at stage 9 stained for $\beta$-Gal (red) and nuclei (DAPI, blue). In the wild type, the expression of $998 / 12$ enhancer trap marker can be observed in the posterior follicle cells from stage 8 onwards. (E) A stage 9 egg chamber containing a $/ g l^{4}$ posterior follicle cell clone marked by the absence of nuclear GFP (green), stained for $\beta$-Gal (red). The posterior follicle cell marker $998 / 12$ is completely absent in the mutant cells, and the oocyte nucleus (arrowhead) has failed to migrate to the dorsal anterior corner. Note that expression of 998/12 is not affected in the posterior follicle cells of the adjoining stage 8 wild-type chamber (arrow). (F) A stage 10 egg chamber in which only part of the posterior follicle cells are mutant and marked by the absence of nuclear GFP (green), stained for $\beta$-Gal (red). Note that $998 / 12$ is still expressed in the remaining wild-type posterior cells (arrow), indicating that Lgl acts cell-autonomously in specifying the posterior follicle cells. In all panels, anterior is to the left and clones are outlined with dots. 
case, Lgl acts cell-autonomously in establishing epithelial polarity through direct regulation of the segregation of the plasma membrane into domains of distinct protein composition. However, this is not the case in the formation of the oocyte polarity associated with the Lgl activities. Instead, the clonal analysis in this report clearly showed that $\mathrm{Lgl}$ in the follicle cells, rather in the oocyte itself, is indispensible for the polarization of the oocyte at mid-oogenesis. Removal of $\mathrm{Lgl}$ function in the posterior follicle cells leads to the non-autonomous oocyte polarity phenotype presumably by perturbing the signaling relay between the oocyte and the overlaying follicle cells. Thus, we have identified a novel function of $\mathrm{Lgl}$ in controlling oocyte polarity which depends on the soma-germline communication.

Initially, $l g l$ was shown to be required in either germline or follicle cells for oogenesis [7]. But subsequent reports implied that $l g l$ function in the germline is not absolutely essential for the egg development, as the females with germline clones homozygous for $l g l^{4}$ produced by the FLP-DFS system can somewhat give rise to mature eggs $[5,11,12,56]$. In our experiments, the absence of $l g l$ gene function in the germline cells never caused either oocyte mispositioning or defective patterning of the oocyte axes. Collectively, these data led us to propose that this gene is differentially required in germline and follicle cells for oogenesis, and the somatic Lgl acts primarily in this developmental process.

We have found that mutations in $l g l$ can perturb the stalk cell differentiation leading to the incorrect positioning of the oocyte. In addition, Lgl may act in the oocyte positioning by affecting the adhesion of the oocyte to the overlying follicle cells at the posterior of the egg chamber. In the relay model described previously [34], the properly formed stalk is required for the posterior localization of the oocyte in its anterior adjacent chamber through inducing the upregulation of the DE-cad adhesion complex in the posterior follicle cells. Indeed, we observed a high percentage of the germline cysts in germarial region 3 with no accumulation of both DE-cad and Arm in the posterior follicle cells when the entire epithelium was mutant for $l g l$. This result suggests that although Lgl may act directly in the up-regulation of the DE-cad adhesion complex, the defects in the adhesion between the oocyte and the posterior follicle cells in the absence of $\mathrm{lgl}$ gene function can be presumably elicited by the failure of the stalk formation. At this time, however, we are not able to distinguish these possibilities.

Proper development of the somatic follicle cell lineages plays a pivotal role in the establishment of both the initial AP asymmetry and the oocyte polarity during oogenesis. In this study, we have found that the posterior follicle cells mutant for $l g l$ do not express the fate-specific marker 998/12, indicating an essential role of $\mathrm{Lgl}$ in the differentiation of those cells. It has been established that the combined and sequential functions of the JAK/STAT, EGFR and Notch signaling control the posterior follicle cell differentiation [53]. Therefore, perturbing one or more of the above signaling pathways can cause the failure of those cells to differentiate normally. We and others have shown that the $l g l$ gene is required in those posterior cells for the mitoticto-endocycle transition, as identified by reduction in the size of the nucleus and presence of phospho-histone3 (PH3) in the mutant clones beyond stage 7 (Figure 5C) [54]. Given that mutations in $l g l$ disrupt Notch localization in the apical side of the posterior follicle cells [54] and that Notch pathway controls the mitotic-to-endocycle transition [40, 54], it seems likely that altered Notch signaling is involved in the defective differentiation and transition in the posterior cells elicited by lack of $l g l$ function. Intriguingly, our preliminary observations revealed that, in contrast to the posterior cells, localized inactivation of $l g l$ function in the anterior/lateral cells does not give rise to either the elevated levels of Fas 3 or the defects in the mitotic-to-endocycle transition (our unpublished data). Although it is yet to be determined which mechanisms underlie the differential requirements of $l g l$ for the specification of the posterior cells versus the anterior/lateral cells, the difference indicated above suggests that loss-of-function mutations in $\mathrm{lgl}$ may perturb the Grk/EGFR signal pathway, leading to loss of the posterior follicle cell differentiation. Analysis of the EGFR signaling activities in $l g l$ mutant posterior cells will help to clarify this question. In the pre-vitellogenic stages, the graded activity of JAK/STAT pathway induced by the polar cells creates a symmetrical prepattern at both poles of each chamber, characterized by induction of the terminal follicle cells [53]. These terminal cells are competent to respond to Grk by adopting a posterior fate. Hence, examination of the expression of the terminal cell markers in the mutant posterior cells will allow us to discern whether the absence of $l g l$ function causes aberrant JAK/STAT signaling linked to the abnormality in the posterior follicle cell specification.

\section{Material and Methods}

\section{Fly strains and genetics}

All strains were maintained and crossed at $25^{\circ} \mathrm{C}$ according to standard procedures unless otherwise stated. Egg chamber stages are according to Spradling [57]. The Canton $S(C S)$ strain was used as wild type. $\lg l^{4}$ is a null allele of $\lg l[2]$ and $\lg l^{t s 3}$ is a hypomorphic temperature-sensitive allele [8].

$\lg l^{t s 3} / C y O, u b i-G F P$ (gift from D. Bilder) females were crossed to $w ; \lg l^{4} F R T 40 A / C y O$, act-GFP (gift from M Sémériva, also used for generating clones) males at permissive temperature $\left(25^{\circ} \mathrm{C}\right)$ to generate $\lg l^{t s 3} / \lg l^{4}$ flies. The transheterozygous female progenies were then shifted to restrictive temperature $\left(29^{\circ} \mathrm{C}\right)$ for 1 or 3 days prior to dissection. 
Homozygous $\lg l^{4}$ clones, marked by the absence of nuclear GFP, were generated by crossing $w ; l g l^{4} F R T 40 A / C y O$, act-GFP to yw122; ubi-GFPnls FRT40A/Cyo (gift from Z Wang). To obtain germline clones, the flies were heat-shocked as third instar larvae at 37 ${ }^{\circ} \mathrm{C}$ for $2 \mathrm{~h}$ on 2 consecutive days. The pupae or newly eclosed adults were heat-shocked at $37^{\circ} \mathrm{C}$ either for $1 \mathrm{~h}$ on 4 successive days or for $2 \mathrm{~h}$ on 2 consecutive days for producing follicle cell clones. Before dissection, all adults were put into fresh food vials for 2-5 days.

The following enhancer trap markers were incorporated into the above fly strains for making $l g l^{4}$ clones: 93F/TM3 (gift from DA Harrison) [18], Kin-lacZ (KZ503, gift from YN Jan) [50] and 998/12/TM3 (gift from D St Johnston) [55].

\section{Antibodies and immunofluorescence}

The following primary antibodies were used in this work: mouse anti-Orb (1:200, DSHB 6H4), rabbit anti-Bib (1:2 000, gift from YN Jan) [42], mouse anti- $\beta$-Gal (1:10, DSHB 40-1a), mouse antiDHC64C (1:100, gift from TS Hays) [58], rabbit anti-Egl (1:4000, gift from R Lehmann) [45], mouse anti-Arm (1:200, DSHB N2 7A1), rat anti-DE-cad (1:200, DSHB DCAD2), rat anti-Stau (1:1 000, gift from Anne Ephrussi), rabbit anti-Osk (1:3 000, gift from A. Ephrussi) [48], mouse anti-Grk (1:200, DSHB 1D12), and mouse anti-Fas3 (1:200, DSHB 7G10). Secondary antibodies conjugated with Alexa Fluor 488, 546, 568 or 647 (Molecular Probes) were used at 1:1000 dilution.

For antibody staining, ovaries were dissected into phosphatebuffered saline (PBS) with $0.1 \%$ bovine serum albumin, fixed in $4 \%$ paraformaldehyde for $30 \mathrm{~min}$ and washed three times with PBST $(0.3 \%$ Triton $\mathrm{X}-100$ in PBS). Then ovaries were permeabilized in PBS with $1 \%$ Triton X-100 for $1 \mathrm{~h}$ at room temperature (RT) followed by a 2 -h incubation in PBST with $10 \%$ normal goat serum. Primary antibodies were incubated with ovaries at $4{ }^{\circ} \mathrm{C}$ overnight. On the following day, ovaries were washed with PBST three times for 20 min each and blocked for $1 \mathrm{~h}$ at RT. Then they were incubated with secondary antibodies at RT for $2 \mathrm{~h}$, and stained with DAPI (BIOMOL Research Laboratories) for $10 \mathrm{~min}$. Finally, ovaries were rinsed three times with PBST and mounted in VECTASHIELD Mounting Medium (Vector Laboratories).

Confocal images were captured on either a Zeiss LSM 510 META laser scanning microscope or a Nikon Eclipse C1si confocal microscope, and processed in Adobe Photoshop.

\section{Acknowledgments}

We thank David Bilder (UC Berkeley, USA), Michel Sémériva (IBDML, France), Douglas A Harrison (University of Kentucky, USA), Yuh Nung Jan (UCSF, USA), Daniel St Johnston (University of Cambridge, UK), Ruth Lehmann (NYU, USA), Thomas S Hays (University of Minnesota, USA), Anne Ephrussi (EMBL, Germany), Zhaohui Wang (CAS, China), the Bloomington Drosophila Stock Center and the Developmental Studies Hybridoma Bank for generously providing us with the fly strains and antibodies. We are also grateful to Qun Sun, Lingzhu Yu, Shunyan Weng, Ling Shen and other members of the Li Lab for technical assistance and discussions. This work was supported by National Basic Research Program of
China (2007CB947300, 2007CB914504), National Natural Science Foundation of China (30470890), the Shanghai Pujiang Program (05PJ14075) and Shanghai Leading Academic Discipline Project (B205).

\section{References}

1 Gateff E, Schneiderman HA. Developmental studies of a new mutant of Drosophila melanogaster: lethal malignant brain tumor (l(2) $\left.g l^{4}\right)$. Am Zool 1967; 7:760.

2 Mechler BM, McGinnis W, Gehring WJ. Molecular cloning of lethal(2)giant larvae, a recessive oncogene of Drosophila melanogaster. EMBO J 1985; 4:1551-1557.

3 Gateff E, Schneiderman HA. Neoplasms in mutant and cultured wild-type tissues of Drosophila. Natl Cancer Inst Monogr 1969; 31:365-397.

4 Gateff E, Schneiderman HA. Developmental capacities of benign and malignant neoplasms of Drosophila. Roux's Arch Dev Biol 1974; 176:23-65.

5 Bilder D, Li M, Perrimon N. Cooperative regulation of cell polarity and growth by Drosophila tumor suppressors. Science 2000; 289:113-116.

6 Bilder D. Epithelial polarity and proliferation control: links from the Drosophila neoplastic tumor suppressors. Genes Dev 2004; 18:1909-1925.

7 Szabad J, Jursnich VA, Bryant PJ. Requirement for cell-proliferation control genes in Drosophila oogenesis. Genetics 1991; 127:525-533.

8 Manfruelli P, Arquier N, Hanratty WP, Semeriva M. The tumor suppressor gene, lethal(2)giant larvae (1(2)g1), is required for cell shape change of epithelial cells during Drosophila development. Development 1996; 122:2283-2294.

9 De Lorenzo C, Strand D, Mechler BM. Requirement of Drosophila l(2)gl function for survival of the germline cells and organization of the follicle cells in a columnar epithelium during oogenesis. Int J Dev Biol 1999; 43:207-217.

10 Arquier N, Perrin L, Manfruelli P, Semeriva M. The Drosophila tumor suppressor gene lethal(2)giant larvae is required for the emission of the Decapentaplegic signal. Development 2001; 128:2209-2220

11 Ohshiro T, Yagami T, Zhang C, Matsuzaki F. Role of cortical tumour-suppressor proteins in asymmetric division of Drosophila neuroblast. Nature 2000; 408:593-596.

12 Peng CY, Manning L, Albertson R, Doe CQ. The tumour-suppressor genes $l g l$ and $d l g$ regulate basal protein targeting in Drosophila neuroblasts. Nature 2000; 408:596-600.

13 Godt D, Tepass U. Drosophila oocyte localization is mediated by differential cadherin-based adhesion. Nature 1998; 395:387391.

14 Gonzalez-Reyes A, St Johnston D. The Drosophila AP axis is polarised by the cadherin-mediated positioning of the oocyte. Development 1998; 125:3635-3644.

15 Gonzalez-Reyes A, St Johnston D. Role of oocyte position in establishment of anterior-posterior polarity in Drosophila. Science 1994; 266:639-642.

16 Gonzalez-Reyes A, Elliott H, St Johnston D. Polarization of both major body axes in Drosophila by gurken-torpedo signalling. Nature 1995; 375:654-658.

17 Roth S, Neuman-Silberberg FS, Barcelo G, Schupbach T. cor- 
nichon and the EGF receptor signaling process are necessary for both anterior-posterior and dorsal-ventral pattern formation in Drosophila. Cell 1995; 81:967-978.

18 Ruohola H, Bremer KA, Baker D, Swedlow JR, Jan LY, Jan YN. Role of neurogenic genes in establishment of follicle cell fate and oocyte polarity during oogenesis in Drosophila. Cell 1991; 66:433-449.

19 Theurkauf WE, Smiley S, Wong ML, Alberts BM. Reorganization of the cytoskeleton during Drosophila oogenesis: implications for axis specification and intercellular transport. Development 1992; 115:923-936.

20 Ray RP, Schupbach T. Intercellular signaling and the polarization of body axes during Drosophila oogenesis. Genes Dev 1996; 10:1711-1723.

21 Berleth T, Burri M, Thoma G, et al. The role of localization of bicoid RNA in organizing the anterior pattern of the Drosophila embryo. EMBOJ 1988; 7:1749-1756.

22 Ephrussi A, Dickinson LK, Lehmann R. Oskar organizes the germ plasm and directs localization of the posterior determinant nanos. Cell 1991; 66:37-50.

23 Ephrussi A, Lehmann R. Induction of germ cell formation by oskar. Nature 1992; 358:387-392.

24 St Johnston D. Moving messages: the intracellular localization of mRNAs. Nat Rev Mol Cell Biol 2005; 6:363-375.

25 Schupbach T. Germ line and soma cooperate during oogenesis to establish the dorsoventral pattern of egg shell and embryo in Drosophila melanogaster. Cell 1987; 49:699-707.

26 Neuman-Silberberg FS, Schupbach T. The Drosophila dorsoventral patterning gene gurken produces a dorsally localized RNA and encodes a TGF alpha-like protein. Cell 1993; 75:165-174.

27 Micklem DR, Dasgupta R, Elliott H, et al. The mago nashi gene is required for the polarisation of the oocyte and the formation of perpendicular axes in Drosophila. Curr Biol 1997; 7:468-478.

28 Deng WM, Ruohola-Baker H. Laminin A is required for follicle cell-oocyte signaling that leads to establishment of the anteriorposterior axis in Drosophila. Curr Biol 2000; 10:683-686.

29 Steinhauer J, Kalderon D. The RNA-binding protein Squid is required for the establishment of anteroposterior polarity in the Drosophila oocyte. Development 2005; 132:5515-5525.

30 Poulton JS, Deng WM. Dystroglycan down-regulation links EGF receptor signaling and anterior-posterior polarity formation in the Drosophila oocyte. Proc Natl Acad Sci USA 2006; 103:1277512780.

31 Steinhauer J, Kalderon D. Microtubule polarity and axis formation in the Drosophila oocyte. Dev Dyn 2006; 235:1455-1468.

32 Margolis J, Spradling A. Identification and behavior of epithelial stem cells in the Drosophila ovary. Development 1995; 121:3797807.

33 Tworoger M, Larkin MK, Bryant Z, Ruohola-Baker H. Mosaic analysis in the Drosophila ovary reveals a common Hedgehoginducible precursor stage for stalk and polar cells. Genetics 1999; 151:739-748.

34 Torres IL, Lopez-Schier H, St Johnston D. A Notch/Delta-dependent relay mechanism establishes anterior-posterior polarity in Drosophila. Dev Cell 2003; 5:547-558.

35 Horne-Badovinac S, Bilder D. Mass transit: epithelial morphogenesis in the Drosophila egg chamber. Dev Dyn 2005; 232:559574.

36 Lantz V, Chang JS, Horabin JI, Bopp D, Schedl P. The Drosophila orb RNA-binding protein is required for the formation of the egg chamber and establishment of polarity. Genes Dev 1994; 8:598-613.

37 Strand D, Raska I, Mechler BM. The Drosophila lethal(2)giant larvae tumor suppressor protein is a component of the cytoskeleton. J Cell Biol 1994; 127:1345-1360.

$38 \mathrm{Xu} \mathrm{T,} \mathrm{Rubin} \mathrm{GM.} \mathrm{Analysis} \mathrm{of} \mathrm{genetic} \mathrm{mosaics} \mathrm{in} \mathrm{developing} \mathrm{and}$ adult Drosophila tissues. Development 1993; 117:1223-1237.

39 Gupta T, Schupbach T. Cct1, a phosphatidylcholine biosynthesis enzyme, is required for Drosophila oogenesis and ovarian morphogenesis. Development 2003; 130:6075-6087.

40 Lopez-Schier H, St Johnston D. Delta signaling from the germ line controls the proliferation and differentiation of the somatic follicle cells during Drosophila oogenesis. Genes Dev 2001; 15:1393-1405.

41 McGregor JR, Xi RW, Harrison DA. JAK signaling is somatically required for follicle cell differentiation in Drosophila. Development 2002; 129:705-717.

42 Larkin MK, Holder K, Yost C, Giniger E, Ruohola-Baker H. Expression of constitutively active Notch arrests follicle cells at a precursor stage during Drosophila oogenesis and disrupts the anterior-posterior axis of the oocyte. Development 1996; 122:3639-3650.

43 Gonzalez-Reyes A, Elliott H, St Johnston D. Oocyte determination and the origin of polarity in Drosophila: the role of the spindle genes. Development 1997; 124:4927-4937.

44 Li M, McGrail M, Serr M, Hays TS. Drosophila cytoplasmic dynein, a microtubule motor that is asymmetrically localized in the oocyte. J Cell Biol 1994; 126:1475-1494.

45 Mach JM, Lehmann R. An Egalitarian-BicaudalD complex is essential for oocyte specification and axis determination in Drosophila. Genes Dev 1997; 11:423-435.

46 St Johnston D, Beuchle D, Nusslein-Volhard C. staufen, a gene required to localize maternal RNAs in the Drosophila egg. Cell 1991; 66:51-63.

47 Neuman-Silberberg FS, Schupbach T. The Drosophila TGFalpha-like protein Gurken: expression and cellular localization during Drosophila oogenesis. Mech Dev 1996; 59:105-113.

48 Vanzo NF, Ephrussi A. Oskar anchoring restricts pole plasm formation to the posterior of the Drosophila oocyte. Development 2002; 129:3705-3714.

49 Roth S. The origin of dorsoventral polarity in Drosophila. Philos Trans R Soc Lond B Biol Sci 2003; 358:1317-1329.

50 Clark I, Giniger E, Ruohola-Baker H, Jan LY, Jan YN. Transient posterior localization of a kinesin fusion protein reflects anteroposterior polarity of the Drosophila oocyte. Curr Biol 1994; 4:289-300.

51 Larkin MK, Deng WM, Holder K, Tworoger M, Clegg N, Ruohola-Baker H. Role of Notch pathway in terminal follicle cell differentiation during Drosophila oogenesis. Dev Genes Evol 1999; 209:301-311.

52 Zhang Y, Kalderon D. Regulation of cell proliferation and patterning in Drosophila oogenesis by Hedgehog signaling. Development 2000; 127:2165-2176.

53 Xi RW, McGregor JR, Harrison DA. A gradient of JAK pathway activity patterns the anterior-posterior axis of the follicular epithelium. Dev Cell 2003; 4:167-177.

54 Deng WM, Althauser C, Ruohola-Baker H. Notch-Delta signaling induces a transition from mitotic cell cycle to endocycle in 
Drosophila follicle cells. Development 2001; 128:4737-4746.

55 Gonzalez-Reyes A, St Johnston D. Patterning of the follicle cell epithelium along the anterior-posterior axis during Drosophila oogenesis. Development 1998; 125:2837-2846.

56 Tanentzapf G, Tepass U. Interactions between the crumbs, lethal giant larvae and bazooka pathways in epithelial polarization. Nat Cell Biol 2003; 5:46-52.

57 Spradling AC. Development genetics of oogenesis. In: Bate M,
Martinez-Arias A, eds. The development of Drosophila melanogaster. Cold Spring Harbor: Cold Spring Harbor Laboratory Press, 1993:1-70.

58 McGrail M, Hays TS. The microtubule motor cytoplasmic dynein is required for spindle orientation during germline cell divisions and oocyte differentiation in Drosophila. Development 1997; 124:2409-2419. 